\title{
Rythmes du vivant et rythme de vie structurent notre rapport au temps $^{1}$
}

\author{
Albert GOLDBETER \\ Unité de Chronobiologie théorique \\ Faculté des Sciences \\ Université Libre de Bruxelles (ULB)
}

\section{Ces rythmes qui nous accompagnent}

La vie est un réseau de rythmes. Pour s'en convaincre il suffit de citer certains d'entre eux qui jouent des rôles majeurs dans de multiples aspects de notre physiologie: rythme cardiaque, rythme de la respiration, rythmes qui interviennent à des niveaux multiples dans la reproduction, rythmes des contractions intestinales ou utérines, rythmes neuronaux et cérébraux, et rythmes circadiens qui régissent le cycle veille-sommeil ainsi que le métabolisme et la nutrition, sans compter les rythmes grâce auxquels nos cellules se divisent. Sans ces rythmes nous ne pourrions respirer, nous reproduire, nous nourrir, nous développer, détecter et traiter nos sensations, penser, ni vivre.

\section{Pourquoi tant de rythmes?}

On connaît en chimie un certain nombre de réactions oscillantes, dont le prototype demeure la réaction de Belousov-Zhabotinsky, du nom des deux chercheurs russes qui l'ont caractérisée il y a plus d'un demi-siècle. L'essor des études des réactions oscillantes à la fin des années 1960 doit beaucoup aux travaux d'Ilya Prigogine qui montra que les rythmes représentent un processus d'auto-organisation temporelle, une "structure dissipative », qui s'établit loin de l'équilibre, quand un état stationnaire devient instable. L'instabilité provient de cinétiques non linéaires associées à des processus d'auto-amplification comme l'autocatalyse (l'effet « boule de neige » connu aussi sous le nom de rétroaction positive, ou " feedback » positif), ou à des rétroactions négatives. De tels processus de régulation sont fort répandus en biologie, car ils permettent d'optimiser la gestion des ressources cellulaires, raison pour laquelle ils ont été sélectionnés au cours de l'évolution. La multiplicité des rythmes du vivant résulte ainsi de la profusion d'interactions régulatrices régissant la dynamique des organismes, des niveaux cellulaire et supra-cellulaire à celui des populations. Ce vaste domaine peut être étendu pour inclure des rythmes à composante psychologique, comme le montre l'exemple des troubles bipolaires. Nous survolerons brièvement ci-dessous les principaux rythmes du vivant, en renvoyant à un livre (Goldbeter, 2010) pour les détails et les références bibliographiques.

\footnotetext{
${ }^{1}$ Ce texte s'inspire, de manière condensée, d'un article cité en référence (Goldbeter, 2016).
} 


\section{Bulletin de la Société Royale des Sciences de Liège [En ligne],}

Vol. 85, 2016, Actes de colloques, Le temps , p. 29 - 37

\section{Des rythmes contrôlent nos principales fonctions physiologiques}

Le battement cardiaque est sans doute le premier rythme qui nous vient à l'esprit. Notre cœur est formé de deux oreillettes et deux ventricules, dont la contraction périodique propulse le sang au sein de l'organisme. On sait, depuis les observations de Harvey il y a plus de trois siècles, que ce rythme est endogène, c'est-à-dire que sa source se trouve dans le cœur même. Il a fallu attendre le début du vingtième siècle pour localiser de manière précise dans le muscle cardiaque l'origine du signal électrique qui, généré de manière répétitive et spontanée, sous-tend la contraction périodique du cœur. C'est dans l'oreillette droite, à proximité de l'arrivée de la veine cave qui ramène le sang non oxygéné vers le cœur, que se trouve le nœud sinusal, un ensemble de cellules capables de générer, de manière spontanée, toutes les secondes environ, le signal électrique qui va se propager aux ventricules en provoquant leur contraction périodique. Si le nœud sinusal ne fonctionne pas correctement, un second nœud, dit auriculo-ventriculaire, situé près de la jonction avec le ventricule droit, prend le relais et produit, avec une fréquence plus faible, le signal périodique menant aux contractions cardiaques. Si chacun de ces deux nœuds vient à faillir dans la genèse des battements du cœur, l'implantation d'un pacemaker artificiel produisant le signal électrique à la fréquence physiologique permet de pallier la défaillance des sources naturelles du rythme cardiaque. A raison d'une contraction par seconde, ou plus fréquemment en cas d'effort physique ou de tension de nature psychologique, le cœur peut battre spontanément de 2 à 3 milliards de fois au cours de la vie. Il s'agit donc d'un oscillateur biologique extrêmement robuste, même si des troubles du rythme peuvent survenir, allant des plus bénins à la fibrillation cardiaque.

La capacité des neurones à produire des rythmes est certainement l'une de leurs principales caractéristiques. Le cerveau produit des rythmes avec une grande variété de fréquences. L'électroencéphalogramme (EEG), qui mesure l'activité électrique globale du cerveau, met en lumière la multiplicité des rythmes cérébraux dont la fréquence varie selon l'aire d'activité, le moment, et l'état de conscience. En phase d'éveil l'activité cérébrale est dominée par les ondes alpha et bêta, dont les fréquences sont de l'ordre de 10 et 15 cycles par seconde - c'est-à-dire 10 et $15 \mathrm{Herz}(\mathrm{Hz})$ - , respectivement. Les ondes thêta, plus lentes, d'une fréquence de 4 à 8 cycles par seconde, surviennent lors de l'endormissement. Le sommeil profond est dominé par les ondes delta, de grande amplitude, et de fréquence plus lente encore, de l'ordre de 1 à 4 cycles par seconde. Lors du sommeil paradoxal, qui correspond à la phase du rêve, le cerveau retrouve une activité rapide dans la gamme des ondes bêta. Enfin, des ondes gamma, d'une fréquence de 30 à $60 \mathrm{~Hz}$, accompagnent les processus cognitifs. Alors qu'un neurone isolé peut déjà présenter un comportement périodique, des rythmes peuvent également résulter des interactions, excitatrices ou inhibitrices, entre cellules faisant partie d'un même réseau neuronal. Ainsi (voir ci-dessous), un réseau neuronal oscillant sous-tend le rythme de la respiration. 


\section{Bulletin de la Société Royale des Sciences de Liège [En ligne], \\ Vol. 85, 2016, Actes de colloques, Le temps , p. 29 - 37}

Le cycle veille-sommeil est contrôlé par l'horloge circadienne, d'une période proche de 24 heures, qui permet aux organismes de s'adapter à l'alternance du jour et de la nuit. La phase de sommeil est elle-même caractérisée par une organisation cyclique, de période plus courte, entre différents stades correspondant à différents types d'activité corticale mesurée par l'électroencéphalogramme. On distingue ainsi quatre phases de sommeil lent, dont deux (1 et 2) de sommeil léger, et deux autres (3 et 4) de sommeil profond. Le sujet qui s'endort passe successivement des phases 1 à 4 puis 4 à 1 ; ce cycle se conclut par une brève phase de sommeil paradoxal, d'une durée de quelque vingt minutes, avant qu'un nouveau cycle de sommeil lent ne commence. Ces cycles se répètent généralement 4 à 5 fois au cours d'une nuit de sommeil, avec une périodicité proche de 90 minutes chez l'homme.

Les oscillations corticales dans la gamme de fréquence proche de $40 \mathrm{~Hz}$, c'est-à-dire dans la gamme des ondes gamma, représentent le substrat neurophysiologique qui sous-tend la perception des stimuli sensoriels, par exemple, olfactifs ou visuels. Ces oscillations paraissent également liées aux processus d'intégration cognitive. Elles surviennent en effet au cours de tâches impliquant divers modes de perception ou d'activité mentale. Pour de nombreux chercheurs, les propriétés rythmiques des neurones sont les piliers sur lesquels s'appuient les bases neurophysiologiques de la conscience.

De même que notre cœur bat, de manière répétitive, spontanément, nous respirons périodiquement, sans devoir y penser. L'automaticité du rythme respiratoire provient d'un réseau neuronal situé dans la moelle épinière. Des études qui se poursuivent depuis une trentaine d'années ont établi que des neurones oscillants, initiateurs de la phase d'inspiration, se trouvent dans une structure neuronale du bulbe rachidien appelée «complexe (pré-) Bötzinger ». Un second oscillateur neuronal, le « groupe respiratoire parafacial », commande la phase d'expiration. Le couplage entre les deux oscillateurs responsables, respectivement, des phases d'inspiration et d'expiration produit le rythme respiratoire.

Les ondes de contraction intestinale jouent un rôle essentiel dans la fonction digestive. Ces ondes sont produites par des oscillateurs qui ne sont autres que les cellules interstitielles décrites dès la fin du $19^{\text {ème }}$ siècle par Ramon y Cajal, au sein du tissu intestinal. Ces cellules produisent des ondes électriques lentes, à raison de quelque dix pulsations par minute, qui sous-tendent la contraction donnant naissance à l'onde intestinale.

De multiples rythmes sont associés, à divers niveaux, à la fonction de reproduction. Le spermatozoïde qui fusionne avec un ovule déclenche des oscillations de calcium, d'une période de quelques minutes, qui vont permettre à l'ovule fécondé de se diviser et de progresser sur le chemin qui mène de l'embryon à l'organisme adulte. Le cycle ovarien ou cycle menstruel, d'une durée de quelque 28 jours, représente un autre rythme marquant de la reproduction. Au cours de ce cycle, l'ovulation est suscitée par des signaux hormonaux de nature pulsatile. Ainsi, l'hormone GnRH est sécrétée par l'hypothalamus à raison d'une pulsation par heure. Seule cette fréquence physiologique permet la sécrétion pulsatile des hormones gonadotropes LH et FSH à des niveaux capables d'induire l'ovulation. La plupart 


\section{Bulletin de la Société Royale des Sciences de Liège [En ligne], \\ Vol. 85, 2016, Actes de colloques, Le temps , p. 29 - 37}

des hormones, y compris celles impliquées dans des processus physiologiques autres que la reproduction, sont sécrétées de manière pulsatile, en plus de variations au cours de la journée. $\mathrm{Au}$ terme même du processus de reproduction, l'accouchement est déclenché par des contractions utérines qui font intervenir, elles aussi, des oscillations de calcium.

Pour qu'un ovule fécondé se développe il doit pouvoir se diviser jusqu'à la formation d'un organisme adulte. Le cycle cellulaire, qui conduit d'une division à la suivante, est régi par un ensemble de réactions biochimiques dont l'enchaînement se répète spontanément, de manière ordonnée. L'ensemble des réactions impliquées dans le cycle cellulaire s'autoorganise dans le temps sous forme d'oscillations entretenues. La transition entre état de quiescence (arrêt de la division) et prolifération cellulaire, due à des divisions répétées, peut être vue comme une transition entre un état stationnaire stable, correspondant à l'arrêt du cycle cellulaire, et un régime d'oscillations entretenues correspondant à une prolifération cellulaire due à des divisions répétées au cours du temps.

\section{L'horloge circadienne}

Les exemples évoqués ci-dessus montrent que les rythmes se rencontrent à tous les niveaux de l'organisation biologique, avec des périodes couvrant plus d'une douzaine d'ordres de grandeur (Goldbeter, 2010), de la fraction de seconde pour les rythmes musculaires ou neuronaux à quelques heures pour les sécrétions d'hormones, sans compter les rythmes annuels caractérisant la floraison des plantes, ou les migrations et la reproduction de nombre d'espèces animales. Des rythmes de périodes plus longues sont observés pour les oscillations prédateurs-proies en écologie, ou la récurrence de certaines épidémies avec des périodicités d'une ou de plusieurs années.

Quand on pense aux horloges biologiques, les premières qui viennent à l'esprit sont toutefois celles qui permettent aux organismes de s'adapter à la caractéristique la plus marquante de la vie sur Terre : l'alternance du jour et de la nuit en 24 heures. Le terme « circadien » (du latin circa, « environ», et dies, «jour ») est utilisé depuis les années 1960 pour caractériser les rythmes biologiques qui se produisent au sein des organismes vivants, y compris certaines espèces de bactéries, avec une période proche de - sans être exactement égale - à 24 heures. Les expériences menées au XVIIIème siècle par un astronome français, Jean-Jacques d'Ortous de Mairan, ont montré que les mouvements des feuilles d'une plante au cours de la journée persistent en obscurité constante : ces rythmes circadiens ont donc une origine endogène, c'est-à-dire qu'ils trouvent leur origine dans des mécanismes internes à la plante et ne résultent pas d'un couplage passif avec l'alternance du jour et de la nuit.

Les connaissances sur le mécanisme moléculaire et cellulaire des rythmes circadiens ont beaucoup progressé au cours des vingt dernières années. On sait à présent qu'au sein de chaque cellule un réseau de gènes oscillant sous-tend le fonctionnement de l'horloge circadienne. Le comportement périodique est fondé sur l'autorégulation négative de certains de ces gènes, en particulier les gènes Per et Cry codant pour les protéines PER et CRY. 
Celles-ci forment un complexe qui, dans le noyau cellulaire, inhibe de manière indirecte l'expression de ces gènes. Cette régulation négative conduit, dans des conditions précises, à l'apparition d'oscillations entretenues d'une période proche de 24 heures. Chez les mammifères, y compris l'homme, l'horloge circadienne principale se trouve dans les noyaux suprachiasmatiques de l'hypothalamus, lesquels sont contrôlés par la lumière, mais des horloges périphériques de même période, possédant leur propre phase, existent dans chaque tissu de l'organisme.

La principale propriété de l'horloge circadienne est d'être entraînée par le cycle lumièreobscurité. Certains des processus biochimiques au sein du mécanisme de l'horloge circadienne sont sensibles à la lumière. Ainsi, la vitesse d'expression du gène Per chez les mammifères augmente au cours de la phase lumineuse. En raison de ce couplage avec le cycle lumière-obscurité, la période de l'horloge circadienne passe d'une valeur proche de 24 heures — période du rythme en libre cours, c'est-à-dire en conditions de lumière ou d'obscurité constante - à 24 heures exactement. Cette propriété dénote l'entraînement de l'horloge circadienne par le cycle lumière-obscurité. L'entraînement permet aux organismes d'être en phase avec l'alternance périodique du jour et de la nuit.

Un syndrome remarquable lié à l'horloge circadienne a été identifié il y a une quinzaine d'années (Toh et al., 2001). Dans une famille étudiée aux Etats-Unis sur plusieurs générations, certains membres de la famille tombent de sommeil aux alentours de $19 \mathrm{~h} 30$ et se réveillent vers $4 \mathrm{~h} 30 \mathrm{du}$ matin. La durée du sommeil est donc dans la moyenne, mais la phase du sommeil est avancée de plusieurs heures. C'est la raison pour laquelle ce syndrome est appelé Syndrome familial d'avance de phase du cycle du sommeil. De manière remarquable, les auteurs ont pu caractériser l'origine génétique de ce syndrome : il s'agit d'une mutation du gène Per2, un gène de l'horloge circadienne codant pour l'une des trois protéines PER présentes chez l'homme. La mutation de la protéine PER2 a pour effet de diminuer la période autonome de l'horloge. Cette diminution de période n'empêche pas l'entraînement de l'horloge circadienne par le cycle lumière-obscurité, mais la phase de l'horloge est avancée de plusieurs heures lors de son accrochage au cycle externe.

\section{Effets d'une perturbation de l'horloge circadienne}

Des travaux récents ont porté sur les effets d'une perturbation brève ou prolongée de l'horloge circadienne. Le décalage horaire, connu sous le nom de jet lag, lié au passage à travers plusieurs fuseaux horaires lors de voyages en avion, se résorbe généralement après quelques jours, temps mis par l'horloge circadienne pour se recaler sur le cycle lumière-obscurité à destination. Plus problématiques peuvent s'avérer les perturbations majeures de l'horloge circadienne, comme le montrent des études chez l'animal et des recherches épidémiologiques chez l'homme. Chez la souris, l'horloge circadienne peut être supprimée par lésion des noyaux suprachiasmatiques ou perturbée de manière permanente par un décalage horaire chronique dans lequel l'animal est soumis à un cycle de $12 \mathrm{~h}$ de lumière suivi de $12 \mathrm{~h}$ d'obscurité, avec une avance de $8 \mathrm{~h}$ de ce cycle effectuée tous les deux jours en réduisant la 
durée de la phase d'obscurité de $12 \mathrm{~h}$ à $4 \mathrm{~h}$. La croissance de tumeurs est accélérée par les deux types de perturbation (Filipski et al., 2002, 2009). Ces études montrent, parmi d'autres, qu'une horloge circadienne fonctionnelle exerce un effet protecteur par rapport à la survenue de cancers. Par ailleurs les études épidémiologiques chez l'homme ont montré que le travail de nuit augmente les risques de développer certains types de cancer ou d'autres maladies comme le diabète de type II. Ces résultats sont importants car de 15 à $20 \%$ des personnes actives travaillent de nuit et sont exposées à la lumière pendant ce qui devrait être leur phase de sommeil. Les résultats de ces études chez l'animal et chez l'homme ont conduit le Centre International de Recherches sur le Cancer, à Lyon, de conclure aux risques probablement cancérogènes du travail de nuit chez l'homme (Straif et al., 2007).

\section{Communications intercellulaires par signaux pulsatiles}

Parmi les rythmes biologiques, les communications intercellulaires de nature pulsatile méritent un intérêt particulier car elles illustrent le rapport étroit entre les rythmes et le temps. Deux exemples sont riches d'enseignements: celui de l'agrégation des amibes sociales et le codage par fréquence des signaux hormonaux (pour une liste de références et un traitement plus détaillé, voir Goldbeter (2010), chapitres 4 et 5).

Les amibes de l'espèce Dictyostelium sont dites sociales, car elles sont capables de passer d'un stade unicellulaire à un stade multicellulaire suite à une carence en nourriture. Les amibes s'agrègent alors en réponse à des signaux chimiques émis de manière pulsatile, avec une période de quelque 5 minutes, par des cellules se comportant comme des centres d'agréation. Le signal pulsatile doit être émis à la bonne fréquence pour que l'agrégation survienne : si le signal est émis de manière constante ou toutes les deux minutes, les cellules ne sont pas capables d'y répondre de manière efficace. De même l'agrégation ne peut survenir si le signal chimiotactique (il s'agit d'une molécule connue sous le nom d'AMP cyclique, ou $\mathrm{AMPc}$ ) est émis avec une fréquence plus faible que la fréquence physiologique.

Un rythme cellulaire commande la sécrétion pulsatile du signal chimiotactique d'AMPc toutes les 5 minutes environ. Le mécanisme du rythme repose sur l'auto-activation de la synthèse du signal suite à la liaison de l'AMPc à son récepteur situé sur la face externe de la membrane cellulaire. Cette régulation autocatalytique ne peut produire un rythme que si elle est couplée à une régulation négative limitant l'effet «boule de neige » de l'autoamplification. La régulation négative prend la forme d'une transition du récepteur vers un état inactif en présence d'AMPc. Un tel phénomène, fort répandu dans les communications intercellulaires, est connu sous le nom de « désensibilisation du récepteur ».

Le système de communication par signaux d'AMPc peut être vu comme un mode primitif de communication hormonale. La plupart des hormones sont sécrétées, elles aussi, de manière pulsatile. Ainsi, l'insuline est sécrétée avec une périodicité d'une dizaine de minutes, l'hormone de croissance avec une périodicité de quelques heures, et l'hormone GnRH, comme indiqué plus haut, à raison d'une pulsation de 5 minutes toutes les heures. La 


\section{Bulletin de la Société Royale des Sciences de Liège [En ligne], \\ Vol. 85, 2016, Actes de colloques, Le temps , p. 29 - 37}

fréquence des pulsations de GnRH s'avère déterminante pour la réponse physiologique suscitée par le signal hormonal. Les expériences ont ainsi montré que le signal de GnRH à la fréquence physiologique est seul capable d'induire les niveaux des hormones gonadotropes LH et FSH nécessaires à l'ovulation. Un signal constant de GnRH, de même qu'un signal trop ou pas assez fréquent ne réussit pas à induite les niveaux adéquats de $\mathrm{LH}$ et FSH, ce qui empêche l'ovulation. Ces résultats ont été mis en œuvre depuis une trentaine d'années dans des approches thérapeutiques: chez des patientes rendues stériles par des troubles de sécrétion de GnRH, l'injection d'une forme synthétique de l'hormone est injectée à la fréquence physiologique d'une pulsation par heure, ce qui permet de rétablir les niveaux de LH et FSH requis pour l'ovulation.

\section{Fréquence optimale des signaux pulsatiles}

Dans les deux cas évoqués plus haut, le rythme de sécrétion du signal pulsatile sert à mesurer le temps : le signal n'exerce un effet optimal que s'il est administré à la fréquence adéquate. Ce codage par fréquence repose sur la désensibilisation du récepteur induite par sa stimulation. Lorsque le signal chimique - l'hormone, ou l'AMPc dans le cas des amibes sociales - se lie à son récepteur situé à la surface de la cellule-cible, cette liaison provoque une réponse qui prend souvent la forme d'une synthèse d'un composé qui va agir au sein de la cellule en suscitant, par exemple, la sécrétion d'un neurotransmetteur ou l'expression de certains gènes. Dans une seconde phase, la stimulation induit la transition du récepteur vers un état désensibilisé dans lequel la réponse cellulaire ne peut plus survenir, ou est significativement réduite, en dépit de la présence maintenue du signal extracellulaire.

Pour assurer le retour d'une réponse maximale, il est nécessaire que la stimulation cesse pendant un laps de temps suffisant, afin de permettre au récepteur de se resensibiliser en retournant à son état actif. Ce laps de temps est spécifique; dans le cas des amibes, il est de quelques minutes, et dans le cas de l'hormone GnRH il est de l'ordre d'une heure. Ceci cadre bien avec la période du rythme de communication intercellulaire, qui est proche de 5 minutes pour la sécrétion du signal chimiotactique chez les amibes, et d'une heure pour la sécrétion pulsatile de GnRH par l'hypothalamus.

\section{Accélération du rythme de vie et rythmes du vivant}

Comme nous l'avons vu plus haut, la multiplicité des rythmes représente l'une des caractéristiques marquantes de la vie. Qu'ils nous soient perceptibles ou non, les rythmes jouent des rôles essentiels en physiologie. Dès la conception, quand un spermatozoïde rencontre un ovule et fusionne avec lui, les rythmes s'installent au cours du développement et nous inscrivent dans le temps. Au-delà des rythmes biologiques proprement dits, un autre aspect du lien entre les rythmes et le temps, de nature plus sociétale, mérite d'être évoqué. Il concerne l'expression « rythme de vie », qu'on associe souvent à l'adjectif « effréné ». Peuton établir un lien entre rythme de vie et rythmes du vivant? 
L'accélération de la vie quotidienne due aux avancées technologiques permettant l'immédiateté des communications représente un thème émergent dans nos sociétés modernes. Cette question fait l'objet de nombreux ouvrages, dont la somme présentée par Hartmut Rosa (2010) dans son livre "Accélération. Une critique sociale du temps », en passant par des ouvrages consacrés au règne de l'urgence (Aubert, 2009; Finchelstein, 2011). Des articles se multiplient dans les magazines et journaux. Ainsi, Le Monde Magazine du 28/9/2010 titrait un entretien avec Rosa: "Au secours ! Tout va trop vite ! ». Des colloques sont consacrés à ce problème. Ainsi, le Forum organisé en 2011 par le journal Le Monde au Mans portait sur le thème "Où va le Temps? — la question au futur se conjugue au passé dans le titre du volume publié l'an suivant (Birnbaum, 2012), comme pour mieux souligner que le temps s'est perdu - tandis qu'une réunion se tenait en juin 2016 à Paris sur le thème «Individus hyperconnectés, société accélérée. Tensions et transformations ».

L'emprise croissante de la vitesse des communications et l'instantanéité dans la propagation des informations, de même que la pression des tâches à accomplir sans délai contribuent à l'accélération de nos rythmes de vie. Devant cette accélération et le stress qui lui est associé, Jean Birnbaum, introduisant dans Le Monde des Livres du 11/11/2011 le forum «Où va le Temps? », conclut son article par une exhortation «(...) il s'agit toujours de préserver, au cœur même de la frénésie, un tempo qui s'accorde avec sa propre pulsation comme avec celle d'autrui. » Cette phrase résonne en nous, mais de quelle pulsation intérieure s'agit-il ?

Ce qu'on appelle «rythme de vie » ne désigne pas un rythme au sens d'un processus qui se répète, après un intervalle de temps égal à une période, et qui se reproduit régulièrement, de manière oscillante. Plutôt qu'un processus périodique, le rythme de vie désigne, de manière globale, l'organisation temporelle de notre vie dans le monde. Contrairement aux rythmes du vivant évoqués plus haut, les rythmes de vie n'ont rien de périodique. Ils font plutôt référence au nombre d'actes que l'on pose au cours d'un laps de temps qui se mesure en heures, en jours, en mois, voire en années. Hyperactivité et hyperstimulation, souvent liées à l'hyperconnexion, vont de pair avec l'accélération du rythme de vie (Rosa, 2010). En économie, l'échelle de temps des transactions à haute fréquence gérées par des algorithmes informatiques est de quelques microsecondes. La civilisation du numérique bouleverse de multiples aspects de nos vies et modifie notre rapport au présent et au passé.

L'échelle de temps de nos rythmes de vie est celle de la journée ou de l'année. Si un lien existe entre rythme de vie et rythmes du vivant, il semble naturel que les plus concernés parmi ceux-ci soient les rythmes circadiens. Des études récentes ont montré que le stress lié à l'accélération du rythme de vie affecte l'horloge circadienne (Cuesta et al., 2015 ; Dumbell et al., 2016); il est plausible que d'autres rythmes, comme celui du cœur ou les rythmes cérébraux, soient également impactés.

Les rythmes qui régissent les communications intercellulaires de nature pulsatile, tels qu'on les observe dans l'agrégation des amibes sociales et dans les sécrétions pulsatiles 
d'hormones, possèdent des implications pour les effets d'une accélération de nos rythmes de vie. Comme on l'a vu plus haut, l'hyperstimulation entraîne souvent une chute de la réponse des cellules-cibles: la stimulation constante ou à trop haute fréquence conduit à la désensibilisation du récepteur et à la perte de la réponse qui s'ensuit. Une stimulation pulsatile à la fréquence optimale donne au récepteur le temps de se resensibiliser, ce qui permet à la cellule de continuer à répondre de manière optimale. Il est tentant de transposer cette observation au plan psychologique : pour éviter le stress - voire, dans un cas extrême, le burn-out (Chabot, 2013) - lié à l'hyperstimulation et à l'accélération du rythme de vie, il semble indiqué de réduire les sources de stimulation excessive et de se ménager des plages de récupération en se déconnectant aussi longtemps et dès que cela s'avère possible. La célébration de la lenteur (Sansot, 1998) et l'essor récent de mouvements qui la prônent en de multiples domaines répondent sans nul doute à ce besoin de réduire la pression de l'accélération sur nos vies, afin de se réapproprier le temps.

\section{Références}

Aubert, N. (2009): Le Culte de l'urgence. La société malade du temps. Champs Flammarion, Paris.

Birnbaum, J. (sous la dir. de). (2012): Où est passé le temps ? Gallimard, Folio Essais, Paris.

Chabot, P. (2013) : Global burn-out. Presses universitaires de France, coll. «Perspectives critiques $»$, Paris.

Cuesta, M., Cermakian, N. \& Boivin, D.B. (2015) : Glucocorticoids entrain molecular clock components in human peripheral cells. FASEB Journal 29: 1360- 1370

Dumbell, R., Mateeva, O. \& Oster, H. (2016) : Circadian Clocks, Stress, and Immunity. Frontiers in Endocrinology 7:37.

Filipski, E. et al. (2002): Host circadian clock as a control point in tumor progression. Journal of the Nationall Cancer Institute 94: 690-697.

Filipski, E. et al. (2009): Circadian disruption accelerates liver carcinogenesis in mice. Mutation Research 680: 95-105.

Finchelstein, G. (2011): La Dictature de l'urgence. Fayard, Paris.

Goldbeter, A. (2016) : Rythmes biologiques et rythme de vie : Vers un décrochage ? In : Temps et Systèmes. Cahiers critiques de thérapie familiale et de pratiques de réseaux 56(1): 233-252.

Goldbeter, A. (2010) : La Vie oscillatoire. Au cour des rythmes du vivant. Odile Jacob, Paris.

Rosa, H. (2010): Accélération. Une critique sociale du temps. La Découverte, Paris (rééd. La Découverte Poche, 2013).

Sansot, P. (1998) : Du bon usage de la lenteur. Payot \& Rivages (rééd. Rivage Poche, Petite Bibliothèque, $\mathrm{n}^{\circ} 313$, Paris, 2000).

Straif, K. et al. (2007) : Carcinogenicity of shift-work, painting, and fire-fighting. The Lancet Oncology 8: 1065-1066.

Toh, K.L. et al. (2001) An hPer2 phosphorylation site mutation in familial advanced sleep phase syndrome. Science 291:1040-1043. 